\title{
EDITORIAL
}

\section{Peer review - a part of the process?}

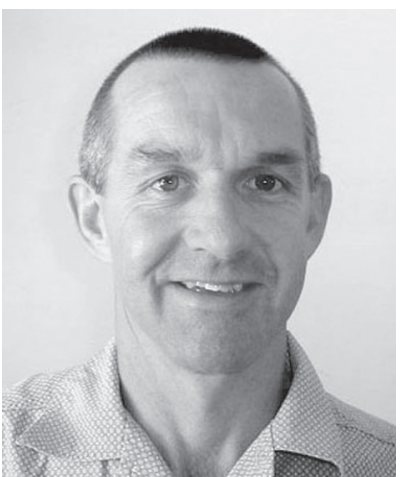

It can be said without doubt that every scientist who has done research and tried to publish a paper in a journal has at some stage been at the receiving end of the peer-review process. There are different forms of peer review associated with publishing research but the most common version is where the editor of the journal chooses reviewers (at least two) who are asked to provide a critique of a paper which has been submitted to the journal. The reviewers are asked to make a recommendation on whether or not the paper should be published. The identity of the authors is not revealed to the reviewers and usually the reviewers are not known to the authors. Reviewers are not paid for their efforts despite the process taking several hours. It is an unwritten code that being asked to review a paper is pay-back for the occasions on which one's own research is reviewed by other unknown scientists. This is the style of review used by the South African Journal of Sports Medicine.

Not everyone supports peer review as it is currently practised. Most of the complaints concern the slow process and the fact that sometimes reviewers might reject a paper because it does not fit into their own research paradigm. Furthermore, the process often fails to detect errors in analysis, ethical problems or scientific fraud. ${ }^{1,2}$ The system has also been accused of blocking innovation and the promotion of new ideas. ${ }^{1}$ The chance of two reviewers of a paper giving identical reports and identifying the same problems with the paper are remote. To counter this problem several reviewers need to be used, but this imposes large time and financial hurdles which most journals find unacceptable. ${ }^{1}$

A recent report concluded that improving peer review '...depends on making its human aspects more humane.
Journals need to ask the right reviewers to review the right articles, help them to do it quickly and thoroughly, make them feel happy to sign their reports, thank them, tell them how they did, and encourage wide recognition of what's too often a thankless task'. ${ }^{1}$

High-quality journals address these points and facilitate the reviewing process by having a large database of reviewers with records of which papers they have reviewed, when they last reviewed a paper, an online tracking system, automated reminders and strict deadlines. Whilst we do not have a sophisticated online tracking system at the SAJSM we do try to enforce strict deadlines for reviewers. However, this process is undergoing change and we hope that by this time next year we will have an online submission with better tracking systems for reviewers.

A problem with the smaller specialist journals such as the SAJSM is the relatively small pool of potential reviewers. We aim to increase this pool by marketing the journal beyond the confines of the members of the South African Sports Medicine Association and if there is sufficient interest we will have workshops on peer review for interested members who may lack experience in reviewing papers.

However, given the acknowledged limitations of peer review it remains the only practical system of quality control presently available. Until a better system evolves or is invented we have to accept and embrace the process and make sure that all the factors which facilitate a smooth reviewing process are controlled.

\author{
Mike Lambert \\ Editor-in-Chief \\ 1. Groves T. Quality and value: How can we get the best out of peer review? \\ Nature 2006; 441: xi - xi. \\ 2. Smith R. Peer review: reform or revolution? BMJ 1997; 315:759-760.
}

